\title{
The most massive AGB stars
}

\author{
Lionel Siess $\dagger$ \\ Institut d'Astronomie et d'Astrophysique, \\ Université libre de Bruxelles, 1050 Bruxelles, Belgium \\ email: siess@astro.ulb.ac.be \\ Centre for Stellar and Planetary Astrophysics, \\ School of Mathematical Sciences, Monash University, Victoria 3800, Australia
}

\begin{abstract}
The general properties of stars in the mass range $7-12 M_{\odot}$, also referred to as super$A G B$ stars, are reviewed and special attention is paid to determine how their mass range depends on the initial metallicity and what fraction of these stars end their life as ONe white dwarfs or explode as electron-capture supernova.
\end{abstract}

Keywords. Stars: AGB and post-AGB, stars: evolution

\section{Introduction}

Stars in the mass range $7-12 M_{\odot}$ experience a peculiar evolution characterized by the off-center ignition of carbon followed by the propagation of a deflagration front toward the center. After the formation of an oxygen-neon (ONe) core, they enter the thermally pulsing SAGB (TP-SAGB) phase during which they experience recurrent thermal instabilities in the helium-burning shell and develop strong winds. The outcome of their evolution is however extremely sensitively to the mass-loss rate and to the efficiency of the third dredge-up during this final evolutionary phase. Depending on these uncertain parameters, the star will either explode as electron-capture supernova (EC-SN) or eject its whole envelope and leave an $\mathrm{ONe}$ white dwarf.

From an historical perspective, the first studies of SAGB stars date back to the 80's where the evolution of helium balls, corresponding to the bare core of stars in the mass range 8-12 $M_{\odot}$, were investigated (Miyaji et al. 1980, Nomoto 1981; 1984, Habets 1986, Baron et al. 1987, Mayle \& Wilson 1988). These early simulations demonstrated the key role of electron capture reactions in triggering the collapse of the ONe core. Different aspects of the complex physics of the explosion (e.g. associated with the role of convective mixing, the impact of Coulomb corrections or the presence of residual ${ }^{12} \mathrm{C}$ in the $\mathrm{ONe}$ core) was thereafter addressed in several papers (e.g. Canal et al. 1992, Gutiérrez et al. 1996, 2005) and hydrodynamical simulations were calculated (Dessart et al. 2003, Kitaura et al. 2006). The first study of the non explosive evolution of SAGB stars from the main sequence up to the thermally pulsing phase was presented in several papers by García-Berro \& Iben (1994), Ritossa et al. (1996), García-Berro et al. (1997), Iben et al. (1997) and Ritossa et al. (1999). Recently new models become available, revisiting the properties of SAGBs at different metallicities and accounting for mass-loss (Gil-Pons et al. 2005,2007; Doherty \& Lattanzio 2006; Siess 2006,2007; Poelarends et al. 2008).

In this paper, the evolutionary features of super-AGB stars are reviewed. A description of the carbon burning phase and subsequent thermally pulsing super-AGB (SAGB) phase is given and, for stars developing the most massive cores, the process leading to the supernova explosion is briefly discussed. In section 3, we address the question of their

$\dagger$ LS is FNRS research associate 
fate, analyzing how the final outcome depends on initial mass, metallicity, mass-loss efficiency and core growth rate.

\section{Main features of super-AGB evolution}

The evolution of the structure up to the end of central helium burning is very similar to that of intermediate mass stars. $\mathrm{H}$ burning takes place in a convective core, the size of which decreases as the increasing He content lowers the central opacity. At H exhaustion, the core contracts and the surface expands leading to the deepening of the convective envelope. During the first dredge-up (1DUP) which only occurs in SAGB stars with $Z>0.001$, the convective zone penetrates into the previously active H-burning region and brings to the surface the ashes of proton burning, i.e. ${ }^{3,4} \mathrm{He},{ }^{13} \mathrm{C}$ and ${ }^{14} \mathrm{~N}$, mainly, leading to a drop in the ${ }^{12} \mathrm{C} /{ }^{13} \mathrm{C}$ ratio. When the central temperature reaches $10^{8} \mathrm{~K} \mathrm{He}$ ignites at the center. A convective core develops and a large amount of ${ }^{12} \mathrm{C}$ is produced by the $3 \alpha$ reactions. When the central He mass fraction drops below $\sim 0.1$, the ${ }^{12} \mathrm{C}(\alpha, \gamma){ }^{16} \mathrm{O}$ reaction becomes the most rapid reaction and a substantial amount of ${ }^{16} \mathrm{O}$ is produced at the expense of ${ }^{12} \mathrm{C}$. As studied by Imbriani et al. (2001) and illustrated in Siess (2007) in the context of SAGB stars, the final $\mathrm{C} / \mathrm{O}$ ratio in the core is very sensitive to the evolution of the core properties near the end of central He burning, and consequently difficult to predict accurately. Typical ${ }^{12} \mathrm{C}$ values range between 0.2 and 0.4 .

\subsection{Carbon burning phase}

When He is depleted at the center, core contraction resumes and as the density increases, neutrinos are further emitted. Energy is removed from the inner regions where the matter becomes degenerate and the temperature inversion moves outward. In low and intermediate mass stars, core contraction releases less energy and stops before the peak temperature reaches the threshold for carbon ignition of $\sim 6 \times 10^{8} \mathrm{~K}$. Then, similarly to the He-flash taking place at the tip of the red giant branch in low mass stars $\left(M \lesssim 2.25 M_{\odot}\right)$, carbon ignites off center in conditions of partial degeneracy (the degeneracy parameter $\eta \sim 2-3$ ). The carbon burning phase proceeds in 2 steps: a flash followed by the propagation of a flame to the center. The flash is more energetic and nuclear luminosities of $10^{6} \lesssim L_{\mathrm{C}} / L_{\odot} \lesssim 2 \times 10^{8}$ can be reached. Because the plasma is partially degenerate, energy accumulates before the pressure starts rising and a strong temperature gradient develops leading to the formation of a first convective zone (Fig. 1). The energy released during the flash induces a large expansion of the burning shells which quenches the instability. As the carbon luminosity $L_{\mathrm{C}}$ decreases, core contraction resumes and the temperature maximum moves inward and increases again. Eventually carbon reignites but the thermodynamical conditions are now somewhat different: the degeneracy is lower $(\eta \sim 1)$ as part of the flash energy was absorbed by the core and the new convective zone that forms develops in a region that was previously occupied by the flash. As a consequence less carbon is made available to power the instability and the resulting luminosity is weaker. The expansion of the core is reduced and convection stays. The star then readjusts toward a steady state configuration (Timmes \& Woosley 1992) where all the energy released by carbon burning is carried away by neutrinos $\left(L_{\nu}=-L_{\mathrm{C}}\right)$. The propagation of the carbon burning front proceeds as a deflagration where ahead of the convective zone, matter is heated up, nuclearly processed and the ashes subsequently engulfed in the convective zone as the peak temperature moves inward where fuel is more abundant. Typical flame speed are of the order of $10^{-3}-10^{-2} \mathrm{~cm} \mathrm{~s}^{-1}$. Finally, when the flame reaches the center, convection disappears and carbon burning proceeds radiatively in a shell surrounding the core. From Fig. 1 we also note that the surface is decoupled 


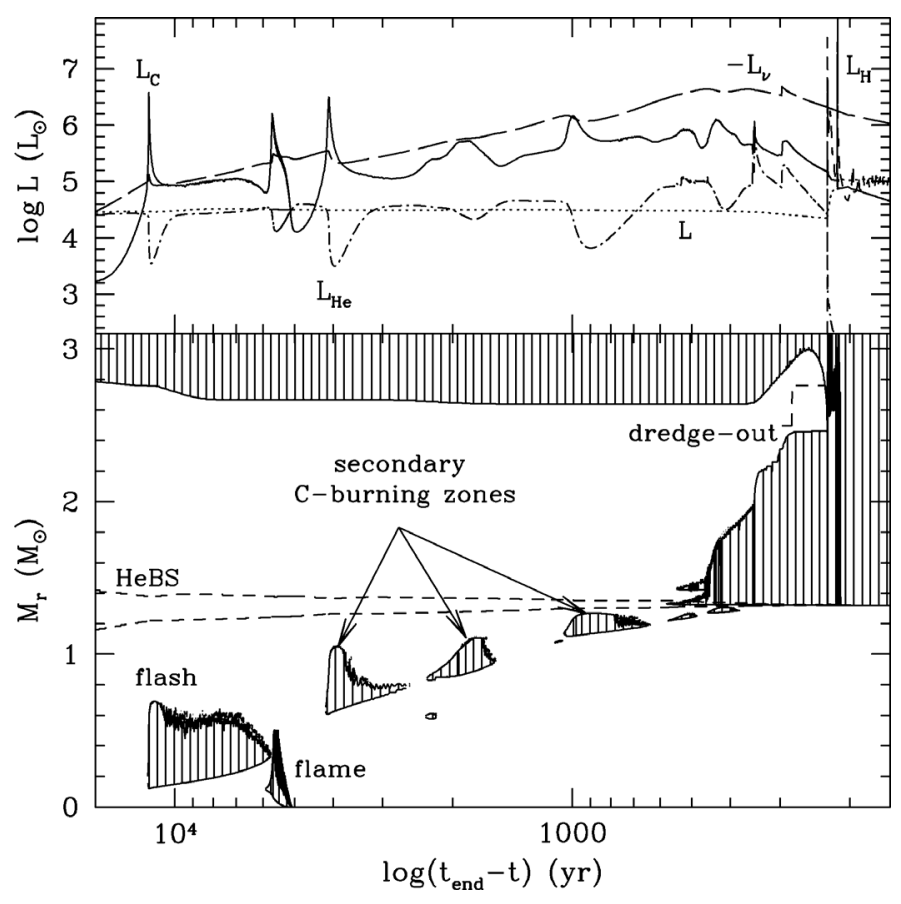

Figure 1. Kippenhahn diagram of a $9.5 M_{\odot} Z=0.001$ star during the carbon burning and dredge-out phases. The hatched areas delineate the convective regions and the time is counted backward from the last computed model. The upper panel displays the evolution of the luminosities associated with $\mathrm{C}\left(L_{\mathrm{C}}\right.$, solid $)$, $\mathrm{H} \quad\left(L_{\mathrm{H}}\right.$, dashed $)$ and $\mathrm{He}$ ( $L_{\mathrm{He}}$, dotted-dashed) burning, with neutrino emission ( $L_{\nu}$, long-dashed) and the surface luminosity $L$ (dotted line) which remains almost constant during this phase.

from the core and is not aware of the structural modifications induced by carbon burning $(L \sim$ constant $)$.

With increasing initial mass, the core temperature is higher and its degeneracy lower. As a consequence the strength of flash and the duration of the flame decrease with increasing mass. Neutrino losses are also weaker in the most massive stars and carbon ignites closer to the center until it reaches the center, in which case the evolution proceeds up to the formation of an iron core in massive stars.

The main nuclear reactions involved in carbon burning are ${ }^{12} \mathrm{C}\left({ }^{12} \mathrm{C}, \mathrm{p}\right)$ and ${ }^{12} \mathrm{C}\left({ }^{12} \mathrm{C}, \alpha\right)$ reactions leading to the production of ${ }^{23} \mathrm{Na}$ and ${ }^{20} \mathrm{Ne} \cdot{ }^{16} \mathrm{O}$ is partially destroyed by $(\alpha, \gamma)$ reactions but at the same time it is replenished by ${ }^{12} \mathrm{C}(\alpha, \gamma)$ so its abundance remains almost unchanged compared to its value at the end of central He burning. The protons and $\alpha$ particles released by C-burning contribute to the production of ${ }^{24} \mathrm{Mg}$ via ${ }^{23} \mathrm{Na}(\mathrm{p}, \gamma)$ and of the ${ }^{25,26} \mathrm{Mg}$ isotopes via $(\alpha, \mathrm{n})$ and $(\alpha, \gamma)$ reactions on ${ }^{22} \mathrm{Ne}$, which was synthesized during central He burning. The activation of the neutron source ${ }^{22} \mathrm{Ne}(\alpha, \mathrm{n})$ may also lead to the production of s-elements. At the end of carbon burning, the core is essentially made of ${ }^{16} \mathrm{O}(\sim 50-70 \%),{ }^{20} \mathrm{Ne}(\sim 15-35 \%)$ followed by ${ }^{23} \mathrm{Na}(\sim 3-5 \%)$ plus some residual ${ }^{24,25,26} \mathrm{Mg}(<3 \%),{ }^{21,22} \mathrm{Ne}$ and ${ }^{27} \mathrm{Al}$.

Interestingly, some unburnt ${ }^{12} \mathrm{C}$ may be left in the core after the passage of the flame. Gutiérrez et al. (2005) showed that if the star enters the electron capture (EC) regime traces of carbon as low as 0.015 (in mass fraction) can induce a thermonuclear runaway by igniting explosive oxygen burning. In these circumstances, the supernova explosion leads to complete disruption of the star as in SNIa. In the core, carbon mass fractions as high as $\sim 0.04$ are found in some of our lowest mass SAGB stars, but they are not expected to follow this fate, provided a binary companion is not influencing their evolution.

\subsection{The second dredge-up}

At the end of core helium burning, the convective envelope moves inward as gravothermal energy flows from the contracting central regions into the envelope. However, because 


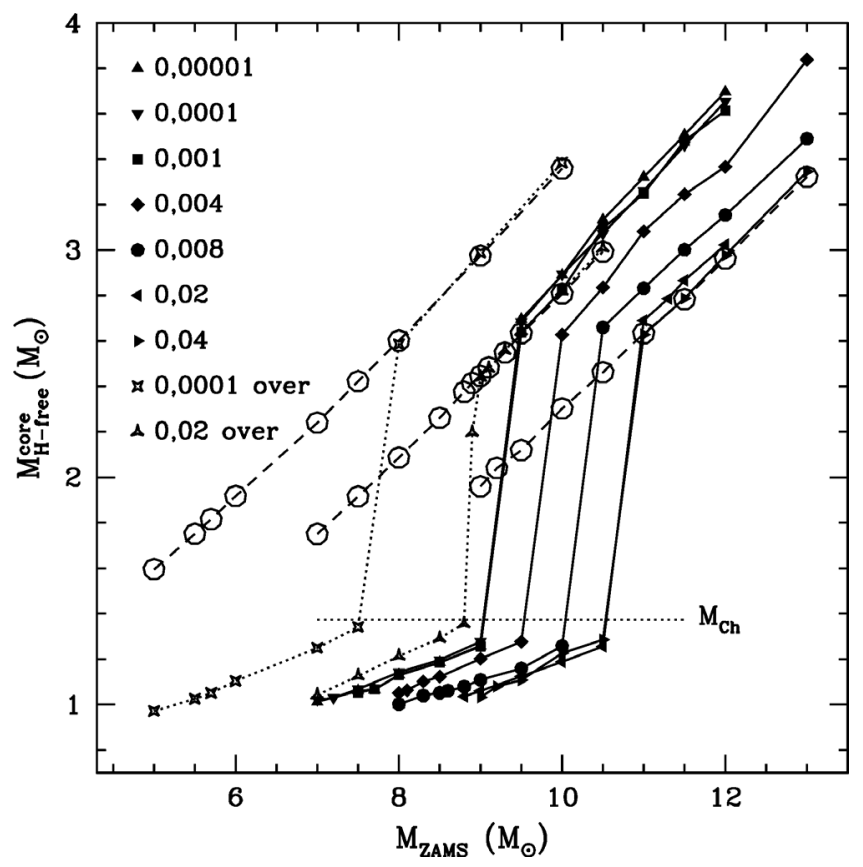

Figure 2. Mass of the $\mathrm{H}$-free core before (dashed line connected by open circles) and after (solid lines) the second dredge-up as a function of the initial mass for various initial compositions. Stars that do not experience the second dredge-up are massive stars that evolve toward core collapse supernova and have $\mathrm{H}$-free cores larger than the Chandrasekhar limit $\mathrm{M}_{\mathrm{Ch}}$.

SAGB stars have more massive cores and evolve faster, carbon may ignite before this process is completed. With increasing mass, the second dredge-up (2DUP) occurs later and it is also able to reach deeper layers. In particular, the convective envelope of SAGB stars penetrates below the former location of the $\mathrm{H}$ burning shell and brings to the surface large amounts of ${ }^{4} \mathrm{He}$ whose mass fraction increases from $\sim 0.25-0.30$ up to $\sim 0.45$. The 2DUP also contributes to enhance ${ }^{13} \mathrm{C}$ and ${ }^{14} \mathrm{~N}$ and decrease the $\mathrm{H},{ }^{12} \mathrm{C}$ and ${ }^{16,18} \mathrm{O}$ surface abundances.

Another characteristic of SAGB evolution is the appearance of the so-called dredge-out phenomenon which takes place at the end of carbon burning when the 2DUP comes to completion (Iben et al. 1997, Siess 2007). In the most massive SAGB stars, the energy transferred from the contracting core to the thick He-rich layers that surrounds the ONe core leads to the development of a convective zone in the He burning shell that grows in mass and moves outwards. Eventually this He-driven convective zone merges with the descending envelope in a relatively energetic event as protons are mixed down to where $\mathrm{He}$ is burnt. This phenomenon produces a substantial increase in ${ }^{4} \mathrm{He}$ and ${ }^{14} \mathrm{~N}$ surface mass fractions.

The 2DUP has another fundamental role: by allowing the envelope to penetrate in the deep stellar interior, it decreases the mass of the H-free core below the Chandrasekhar limit $\left(M_{\mathrm{Ch}}\right)$. This is illustrated in Fig. 2 where the mass coordinate of the H-exhausted core before (open symbols) and after the completion of the 2DUP is displayed. It is interesting to note that the transition between stars that do and do not experience this deep mixing event occurs abruptly, in a very narrow mass interval of $\lesssim 0.5 M_{\odot}$. Massive stars avoid the 2DUP and because their core mass exceeds $M_{\mathrm{Ch}}$ they are able to activate all nuclear reactions up to the formation of an iron core. SAGB stars could thus be defined as the most massive stars that still experience the 2DUP. 


\subsection{The thermally pulsing $S A G B$ phase}

With the decay of carbon burning and the increasing neutrino emission, the contracting core becomes more degenerate. At the same time, the He burning shell (HeBS) thins out and as it approaches the $\mathrm{H}$ rich layers the H-burning shell (HBS) progressively re-ignites.

At this stage of the evolution, the structure of SAGB stars is very similar to that of a lower mass AGB star and the evolution proceeds in an alternate shell burning mode characterized by the development of recurrent convective instabilities in the HeBS. The main difference with "standard" AGB stars resides in the mass and composition of the degenerate core. Because SAGB stars have more massive cores (the minimum mass of an $\mathrm{ONe}$ core is predicted to be $\gtrsim 1.05 M_{\odot}$, Siess 2007 ), the gravitational pull is stronger and the burning shells are more compressed. They are consequently thinner and their temperature is higher as well. The mass extent of a convective pulse is typically less than a few $10^{-4} M_{\odot}$ which is more than one order of magnitude smaller that in AGB stars. Owing to the higher temperature in the pulse, when the convective instability develops, the structure reacts more quickly because of the larger contribution of the radiative pressure $\left(\propto T^{4}\right)$ to the total pressure. The result is that the thermal pulses are weak (the He generated luminosity $L_{\mathrm{He}} \lesssim 10^{6} L_{\odot}$ ) and their duration relatively short $\left(\Delta_{\text {pulse }}<\right.$ few years). The interpulse duration is also reduced to a few hundred years which implies that the SAGB stars will experience a huge number of thermal pulses, as high as several thousands. But the compressional heating of the internal layers does more than impacting the structure, it also significantly affects the surface abundances, as we shall discuss now.

\subsection{Nucleosynthesis}

In all our SAGB models, the temperature at the base of the He-driven convective zone exceeds $3.2 \times 10^{8} \mathrm{~K}$, allowing for the efficient activation of the ${ }^{22} \mathrm{Ne}(\alpha, \mathrm{n})$ neutron source. In principle, this reaction can lead to the synthesis of $s$-process elements but the efficiency of this process may be reduced by the short duration of the neutron irradiation. In addition, because of the large dilution factors involved (the mass of the convective pulse is 4 to 5 orders of magnitude smaller than that of the envelope), the pollution of the surface by third dredge-up (3DUP) episodes is weak and could be hard to detect observationally. It should be noted that He is partially burnt during the thermal pulse and, at the time of the 3DUP, the intershell composition is strongly enriched in ${ }^{22} \mathrm{Ne}$ and ${ }^{25,26} \mathrm{Mg}$ resulting from the $\alpha$-captures reactions on ${ }^{14} \mathrm{~N}$ and ${ }^{22} \mathrm{Ne}$, respectively.

The main signature of SAGB star nucleosynthesis results from the onset of very efficient proton burning at the base of the convective, also called Hot Bottom Burning (HBB). With envelope temperature $\left(T_{\text {env }}\right)$ ranging between $10^{8}$ and $1.4 \times 10^{8} \mathrm{~K}$, all proton burning reactions are activated. The $\mathrm{CN}$ cycle works at equilibrium imposing a ${ }^{12} \mathrm{C} /{ }^{13} \mathrm{C}$ ratio $\sim 4$, the $\mathrm{ON}$ cycle leaks into the NeNa chain and the two isotopes ${ }^{16,18} \mathrm{O}$ are partially destroyed. The NeNa chain efficiently converts ${ }^{22} \mathrm{Ne}$ into ${ }^{23} \mathrm{Na}$ but above $9 \times 10^{7} \mathrm{~K}$, the cycle is broken as $(\mathrm{p}, \gamma)$ reactions onto ${ }^{23} \mathrm{Na}$ become faster that the $(\mathrm{p}, \alpha)$ reactions on this element. ${ }^{24} \mathrm{Mg}$ is rapidly depleted to the benefit of ${ }^{25} \mathrm{Mg}$ and ${ }^{26} \mathrm{Al}_{g}$ is then substantially produced by ${ }^{25} \mathrm{Mg}(\mathrm{p}, \gamma)$. Given the short duration of the TP-SAGB phase $\left(\tau_{\mathrm{SAGB}} \lesssim 10^{5} \mathrm{yr}\right),{ }^{26} \mathrm{Al}_{g}$ does not have time to $\beta$-decay but above $\gtrsim 1.2 \times 10^{8} \mathrm{~K}$ this element suffers heavy proton captures favoring the production of ${ }^{27} \mathrm{Al}$.

To summarize, because HBB is very strong in SAGB stars, the envelope is efficiently processed by proton burning, increasing the surface abundance of ${ }^{14} \mathrm{~N},{ }^{25} \mathrm{Mg}$ and ${ }^{26,27} \mathrm{Al}_{g}$ and imposing ${ }^{12} \mathrm{C} /{ }^{13} \mathrm{C} \sim 4$. 


\subsection{SAGB yields}

The yields of SAGB stars are largely unknown and often not accounted for in chemical evolution models despite the relative weight of this stellar population in regards to the IMF. One of the reasons for this lack of data is the huge amount of CPU time needed to follow their full evolution. Assuming, that a 1D stellar evolution code can simulate about 10 thermal pulses a day, given the fact that the star will experience approximately a thousand instabilities, one won't be able to get the uncertain number before at least 3 months! Furthermore, the determination of yields is subject to large uncertainties associated with the efficiency of the third dredge-up, the nuclear reaction rates and, as we shall see, with the thermodynamical conditions at the base of the convective envelope.

The impact of the 3DUP is the result of a subtle competition between on one hand the weak pollution induced by each mixing episode (the envelope mass is up to $10^{4}-10^{5}$ larger than that of the dredged-up material) and on the other hand on the very large number of thermal pulses. But the problem is more complex and strongly dependent on the initial mass and metallicity. In particular, the imprint of the 3DUP will be stronger at lower metallicity as a consequence of the higher chemical contrast between the intershell and envelope compositions (Siess 2008, in preparation). As a general trend, the 3DUP increases the envelope abundances of ${ }^{12} \mathrm{C},{ }^{14} \mathrm{~N}$ and ${ }^{22} \mathrm{Ne}$. But this material is subsequently processed by HBB, the efficiency of which is primarily controlled by $T_{\text {env }}$. Unfortunately, our poor understanding of convection does not allow us to precisely determine this temperature. Changing the MLT parameter $\alpha$ or using a different convection model has a strong influence on the efficiency of the HBB (Sackmann \& Boothroyd 1991, Ventura \& d'Antona 2005) through the modification of $T_{\text {env }}$. The impact is considerable in SAGB stars and can easily exceed the chemical modifications induced by the presence of the 3DUP (Siess \& Arnould 2008). A change in $T_{\text {env }}$ mostly affects the nuclides involved in the high temperature proton burning chains. In particular, the abundances of ${ }^{17} \mathrm{O},{ }^{22} \mathrm{Ne}$, ${ }^{23} \mathrm{Na},{ }^{24,26} \mathrm{Mg}$ and ${ }^{26} \mathrm{Al}_{g}$ can be substantially altered. For example, a $10 \%$ uncertainty in $T_{\text {env }}$ leads to variations in the ${ }^{26} \mathrm{Al}_{g}$ mass fraction by a factor ranging between 0.3 and 7 (Siess \& Arnould 2008)!

The SAGB yields thus appear to be subject to even larger uncertainties than their lower mass AGB counterparts because of the strong dependence of their surface composition on the efficiency of $\mathrm{HBB}$. Despite these severe limitations, we can reasonably consider SAGB stars as strong contributors of ${ }^{13} \mathrm{C},{ }^{14} \mathrm{~N},{ }^{17} \mathrm{O},{ }^{25} \mathrm{Mg}$ and ${ }^{26} \mathrm{Al}$.

\section{The final fate of super-AGB stars}

\subsection{The mass range of $S A G B$ stars}

The masses of SAGB stars range between $M_{\mathrm{up}}$, the minimum initial mass for (off-center) carbon ignition and $M_{\text {mas }}$ the minimum mass above which the star evolves through all nuclear burning stages up the formation of an iron core. Below $M_{\mathrm{up}}$, the evolution leads to the formation of a $\mathrm{CO}$ white dwarf (WD) and above $M_{\text {mas }}$ the remnant is a neutron star or a black hole depending on the initial mass, composition, mixing and mass-loss rate. The dependence of $M_{\text {up }}$ and $M_{\text {mas }}$ on the initial metallicity is depicted in Fig. 3 for standard models without core overshooting. The decrease in $M_{\mathrm{up}}$ and $M_{\mathrm{mas}}$ with $Z$ is due to opacity effects: with less metals, the opacity drops and the temperature and surface luminosity increase. To cope with the structural changes and higher energy demand, the star develops a bigger core which is more prone to ignite carbon (see Siess 2007 for details). It is important to stress that the values of these transition masses are extremely sensitive to the treatment of mixing at the edge of the convective core, the 
effects becoming critical during central He burning. Taking into account overshooting in the computations increases the size of the core and makes the star behave as if it was more massive. The effect of this extra mixing is significant and shifts the mass range of SAGB stars down by $\sim 2 M_{\odot}$ (e.g. Siess 2007). This can have significant consequences on chemical evolution models given the strong mass dependence of the IMF. As for massive stars, the evolution of SAGB stars is strongly dependent on the adopted treatment of mixing but also and unavoidably on the numerics (Poelarends et al. 2008).

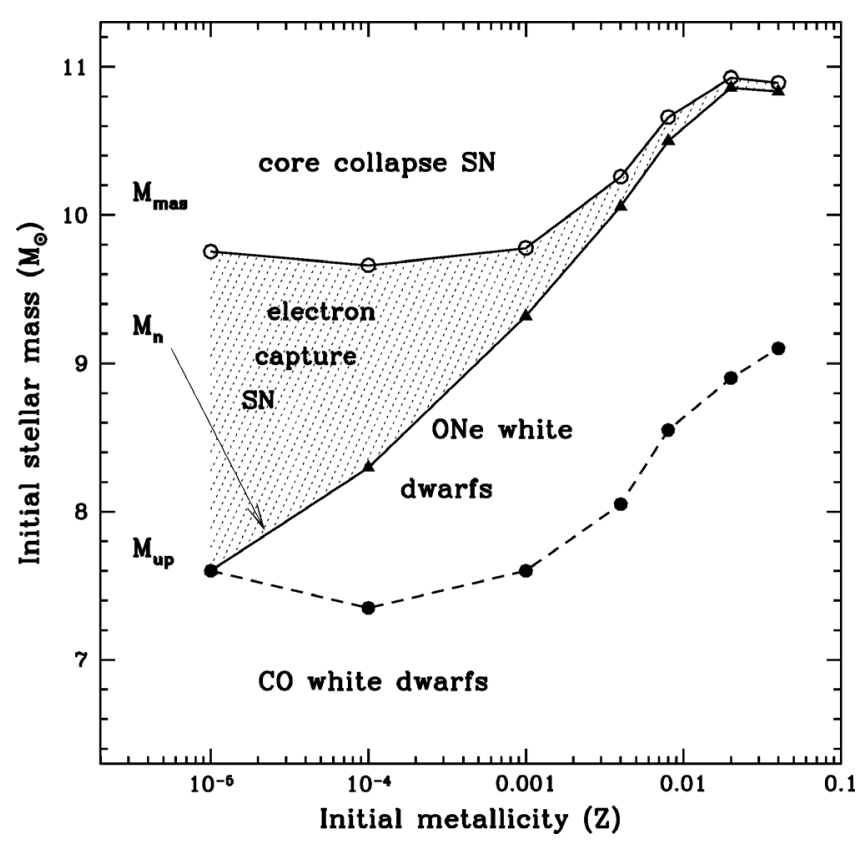

Figure 3. Evolution of the different mass transitions $\left(M_{\mathrm{up}}\right.$, $M_{\mathrm{n}}$ and $\left.M_{\mathrm{m} \text { as }}\right)$ as a function of initial metallicity. Below $M_{\mathrm{up}}$, the remnant is a $\mathrm{CO}$ white dwarf, between $M_{\mathrm{up}}$ and $M_{\mathrm{n}}$ the endpoint of the evolution is the formation of an $\mathrm{ONe}$ WD. Stars more massive than $M_{\text {mas }}$ will proceed through all nuclear burning stages and end their life as core collapse $\mathrm{SNe}$ while stars between $M_{\mathrm{n}}$ and $M_{\mathrm{mas}}$ will experience EC-SN. $M_{\mathrm{n}}$ was computed assuming $\dot{M}_{\text {core }}=5 \times 10^{-7} M_{\odot} \mathrm{yr}^{-1}$ and $\dot{M}_{\text {wind }}=10^{-5} M_{\odot} \mathrm{yr}^{-1}$.

\subsection{The electron capture channel for $S A G B$ stars}

After the completion of the 2DUP, the star enters the thermally pulsing AGB phase during which the mass of the degenerate core increases by the accretion of the ashes of $\mathrm{H}$ and $\mathrm{He}$ shell burning. As the core grows, the central density increases, so does the Fermi energy of the electrons $\varepsilon_{\mathrm{F}}$. When $\rho>10^{9} \mathrm{~g} \mathrm{~cm}^{-3}$ the mass of the core has reached $\sim 1.37 M_{\odot}$ and $\varepsilon_{\mathrm{F}}$ exceeds the threshold energy for electron captures on various elements. These reactions first involve ${ }^{27} \mathrm{Al}$, followed by ${ }^{25} \mathrm{Mg},{ }^{23} \mathrm{Na}$ before operating on the abundant ${ }^{20} \mathrm{Ne}$ (Ritossa et al. 1999). At this time, the structure of the core is highly degenerate and pressure is almost entirely supported by the electrons. Thus, when electrons start being substantially captured by ${ }^{20} \mathrm{Ne}$ the pressure drops and core collapse begins. The rapid contraction of the internal layers leads to oxygen ignition and to the conversion of the matter into a nuclear statistical equilibrium (NSE) mixture. The subsequent electron captures on these NSE elements accelerates the collapse that proceeds until the density of nuclear matter is reached (Nomoto 1987). The equation of state stiffens and the inner collapsing shells bounce on the newly formed neutron star. The so-called prompt bounce shock initially progresses outward but it rapidly $(\sim 1 \mathrm{~ms})$ loses energy by photodissociating and inverting the motion of the infalling shells. It is thanks to the energy deposited by neutrinos that the shock will be revived and the explosion successful (e.g. Dessart et al. 2003, Kitaura et al. 2006, Janka et al. 2008). These explosions are expected to produce sub-luminous type II-P SNe, due to the low H 
envelope mass ( $\lesssim 6-7 M_{\odot}$ ) and small production of ${ }^{56} \mathrm{Ni}$ (e.g. Bethe \& Wilson 1985, Kitaura et al. 2006).

The key point in determining the fate of the SAGB star is whether or not the core mass is able to reach the critical value of $M_{\mathrm{EC}}=1.37 M_{\odot}$. For instance, if the wind is strong or the core growth somehow reduced (e.g. by deep 3DUPs), the envelope is removed before the central density reaches the threshold for electron capture reactions and the remnant is an ONe WD. On the contrary, if the mass-loss rate is weak, the core can grow up to $M_{\mathrm{EC}}$ and a supernova explosion ensues. The fate of the star thus depends on two largely unknown parameters, namely the core growth rate $\dot{M}_{\text {core }}$ and the mass-loss rate $\dot{M}_{\text {wind }}$. The determination of $\dot{M}_{\text {core }}$ is directly related to the efficiency $\lambda$ of the $3 \mathrm{DUP} \dagger$ which depends on our poor understanding of the convective boundaries (see e.g. the contribution of F. Herwig in this proceeding). On the other hand, the knowledge of the mass-loss rate is not better constrained. Since SAGB stars fill the gap between intermediate and massive stars, we have in practice the choice of using mass-loss rate prescriptions that are suited for either stellar class. For typical SAGB stars of $\sim 10^{5} L_{\odot}, \sim 1200 R_{\odot}$ and $T_{\text {eff }} \simeq 3000 \mathrm{~K}$, the mass-loss rate ranges between $5.3 \times 10^{-6} M_{\odot} \mathrm{yr}^{-1}$ and $1.5 \times 10^{-4}$ if one uses the de Jager et al. (1988) or the Vassiliadis \& Wood (1993) prescriptions, respectively. The uncertainty in $\dot{M}_{\text {wind }}$ is further increased by our poor knowledge of its dependence on the metallicity.

The dividing mass between stars that end their life as ONe WD and those that explode as EC-SN is usually referred to as $M_{\mathrm{n}}$ and is determined as follows (see Siess 2007 and Poelarends et al. 2008 for details). For a given set of parameters $\left(\dot{M}_{\text {core }}, \dot{M}_{\text {wind }}\right)$, we can determine for each star the amount of mass $\Delta M_{\text {core }}$ that has been accreted on top of the core during the TP-SAGB phase. From these data, we can then find the initial mass such that, at the end of the evolution when the envelope is removed, the core mass is just equal to the $M_{\mathrm{EC}}$, i.e. we search for $M_{\mathrm{n}}$ satisfying $M_{\text {core }}^{\text {end }}\left(M_{\mathrm{n}}\right)=M_{\text {core }}^{\text {eAGB }}\left(M_{\mathrm{n}}\right)+$ $\Delta M_{\text {core }}\left(\dot{M}_{\text {core }}, \dot{M}_{\text {wind }}\right)=1.37 M_{\odot}$, where $M_{\text {core }}^{\text {eAGB }}$ is the core mass at the beginning of the TP-SAGB phase. If we set $\dot{M}_{\text {core }}=5 \times 10^{-7} M_{\odot} \mathrm{yr}^{-1}$ (which would correspond to a very inefficient 3DUP characterized by $\lambda \simeq 0$ ) and impose $\dot{M}_{\text {wind }}=10^{-5} M_{\odot} \mathrm{yr}^{-1}$ and further assume that $\dot{M}_{\text {wind }}$ has a metallicity dependence of the form $\left(Z / Z_{\odot}\right)^{1 / 2}$ (Kudritzki et al. 1987), we obtain for $M_{\mathrm{n}}$ the curve shown in Fig. 3. With decreasing metallicity, the mass-loss rate is reduced and a larger fraction of SAGB stars is able to enter the EC-SN regime. We also note that at solar metallicity (with our adopted parameters), the mass window for EC-SN is very small, typically less than $\sim 0.5 M_{\odot}$ in width. Conversely, at very low $Z$ (below $\sim 10^{-4}$ ) because of the reduced mass-loss rate, most SAGB stars are expected to follow the EC-SN channel. In this model, the formation of ONe WD is even prevented for $Z \lesssim 10^{-5}$. If we now follow Poelarends et al. (2008) and use a Salpeter IMF to estimate the fraction $f_{\mathrm{EC}}$ of EC-SN out of all the SNe we get the following numbers: at solar metallicity, for $M_{\mathrm{mas}}=10.93 M_{\odot}$ and the previously adopted parameters, we obtain $M_{\mathrm{n}}=10.85 M_{\odot}$ and $f_{\mathrm{EC}}=1 \%$. If instead, we had used a reduced core growth rate of $\dot{M}_{\text {core }}=5 \times 10^{-8} M_{\odot} \mathrm{yr}^{-1}$ (which would have been obtained if $\lambda=0.9$ ), $M_{\mathrm{n}}=10.10 M_{\odot}$ and $f_{\mathrm{EC}}$ rises up to $\sim 10 \%$. Note that these percentages are weakly affected by the consideration of extra-mixing at the core edge which strongly modifies the mass range of SAGB stars $\left(M_{\mathrm{up}}\right.$ and $\left.M_{\mathrm{mas}}\right)$. As illustrated, $f_{\mathrm{EC}}$ strongly depends on the 3DUP efficiency but also on the mass-loss rate. With more realistic values of these parameters, Poelarends et al. (2008) find that the current (i.e. at $Z=Z_{\odot}$ ) fraction of SNe involving a SAGB progenitor ranges between $\sim 4-20 \%$.

$\dagger$ By definition and provided the rate of advance of the $\mathrm{H}$ burning shell is not affected by the 3 DUP, $\dot{M}_{\text {core }}(\lambda)=[1-\lambda] \times \dot{M}_{\text {core }}(\lambda=0)$ 


\section{Conclusion}

This overview of SAGB stars has illustrated the wealth of physical processes that pace the evolution of this peculiar stellar population, from the off-center ignition of carbon up to the hydrodynamical phase of the supernova explosion. Among other interesting aspects that were not addressed in this review and which are of fundamental interest is the development of the convective URCA process that starts with the activation of the electron capture reactions (e.g. Ritossa et al. 1999). This process is poorly understood and has not yet been fully investigated in our context. It is however of great importance since it sets the conditions for the explosion.

Even if the direct observation of an evolved TP-SAGB star has yet to be confirmed, there are several evidences of their existence. The detection of ONe WD in novae systems (e.g. José \& Hernanz 1998) provides certainly the best and most studied observational counterpart of SAGB stars. Actually, ONe WD are very numerous and may be at the center of $\sim 1 / 3$ of all observed novae (Gil-Pons et al. 2003). It is also worth emphasizing that the WD mass distribution (e.g. Liebert et al. 2005) shows an extended tail towards higher masses, between $1 \lesssim M_{\mathrm{WD}} / M_{\odot} \lesssim 1.2$ which perfectly fits within the mass range of ONe cores. More recently, the progenitor mass of several sub-luminous type II-P supernovae (e.g. Hendry et al. 2005, 2006, Maund et al. 2005, Li et al. 2007) was estimated. Most interestingly, these studies reveal that these explosions were triggered by stars of $\sim 6-13 M_{\odot}$, supporting the existence of an EC-SN channel for SAGB stars. Such explosions may also produce neutron stars with low-velocity natal kicks (e.g. Podsiadlowski et al. 2004) and be responsible for the formation of high-mass X-ray binaries with long orbital periods and low eccentricities (Pfahl et al. 2002). As we have shown previously, because of their relatively high contribution to the IMF, SAGB stars may account for a significant fraction of the currently observed SNII. For the same reason they represent a key ingredient of chemical evolution models and are expected to be strong providers of ${ }^{13} \mathrm{C},{ }^{14} \mathrm{~N},{ }^{17} \mathrm{O},{ }^{25} \mathrm{Mg}$ and ${ }^{26} \mathrm{Al}$ as a result of very efficient HBB. In particular, their impact may explain the ${ }^{13} \mathrm{C}$ and ${ }^{14} \mathrm{~N}$ enrichments of the interstellar medium observed at low metallicities (Chiappini et al. 2005, 2008). From a nucleosynthesis perspective, SAGBs have long been ascribed to be the site of the production of $r$-process elements but this idea is apparently in contradiction with recent consistent hydrodynamical simulations (Hoffman et al. 2008) despite previous claims (e.g. Ning et al. 2007).

It must be emphasized that all these mass transitions are highly uncertain and strongly dependent on numerous and badly constrained factors such as the mass-loss rate, the properties of the 3DUP, the presence of extra-mixing or the numerics (Eldridge et al. 2004, Siess 2007, Poelarends et al. 2008). More detailed models of the evolution of SAGB will help clarify these issues and ascertain their role in the chemical evolution of galaxies.

\section{Acknowledgements}

The author thanks the organisers for financial support and is most grateful to John Lattanzio for his comments and generous hospitality during the writing of this proceeding. This research was supported under Australian Research Council's Discovery Projects funding scheme (project number DP0877317).

\section{References}

Baron, E., Cooperstein, J., \& Kahana, S. 1987, ApJ, 320, 300

Bethem, H. A. \& Wilson, J. R. 1985, ApJ, 295, 14

Canal, R., Isern, J, \& Labay J. 1992, ApJ, 398, 49

Chiappini, C., Ekstrm, S., Meynet, G. et al. 2008, A\&A, 479, L9 
Chiappini, C., Matteucci, F., \& Ballero, S. K. 2005, A\&A, 437, 429

de Jager, C., Nieuwenhuijzen, H., \& van der Hucht K. A. 1988, A\&AS, 72, 259

Dessart, L., Burrows, A., Ott, C. D., et al. 2003, ApJ, 644, 1063

Doherty, C. L. \& Lattanzio, J. C. 2006, MmSAI 77, 828

Eldridge, J. J. \& Tout, C. A. 2004, MNRAS, 353, 87

Gil-Pons, P., García-Berro, E., José, J., Hernanz, M., \& Truran, J. W. 2003, A\&A, 407, 1021

Gil-Pons, P., Suda, T., Fujimoto, M. Y. \& García-Berro, E. 2005, A\&A, 433, 1037

Gil-Pons, P., Gutiérrez, J., \& García-Berro, E. 2007, A\&A, 464, 667

García-Berro, E. \& Iben, I. 1994, ApJ, 434, 306

García-Berro, E., Ritossa, C., \& Iben, I. 1997, ApJ, 485, 765

Gutiérrez, J., Canal, R., \& García-Berro, E. 2005, A\&A, 435, 231

Gutiérrez, J., García-Berro, E., Iben, I., et al. 1996, ApJ, 459, 701

Habets, G. M. H. J. 1986, A\&A, 167, 61 ApJ, 489, 772

Hendry, M. A., Smartt, S. J., Maund, J. R., et al. 2005, MNRAS, 359, 906

Hendry, M. A., Smartt, S. J., Crockett, R. M., et al. 2006, MNRAS, 369, 1303

Hoffman, R. D., Mller, B., \& Janka, H.-T 2008, ApJ, 676, L127

Iben, I., Ritossa, C., \& García-Berro, E. 1997,

Imbriani, G., Limongi, M., Gialanella, L., Straniero, O., \& Chieffi, A. 2001, ApJ, 558, 903

Janka, H.-T, Mller, B., Kitaura, F. S., \& Buras, R. 2008, A\&A, in press

José, J. \& Hernanz, M. 1998, ApJ, 494, 680

Kitaura, F. S., Janka, H.-Th., \& Hillebrandt, W. 2006, A\&A, 450, 345

Kudritzki, R. P., Pauldrach, A., \& Puls, J. 1987, A\&A, 173, 293

Li, W., Wang, X., Van Dyk, S. D., et al. 2007, ApJ, 661, 1013

Liebert, J., Bergeron, P., \& Holberg, J. B. 2005, ApJS, 156, L47

Maund, J. R., Smartt, S. J., \& Danziger, I. J. 2005, MNRAS, 364, L33

Mayle, R. \& Wilson, J. R. 1988, ApJ, 334, 909

Miyaji, S., Nomoto, K., Yokoi, K., \& Sugimoto, D. 1980, Publ. Astron. Soc. Japan, 32, 303

Ning, H., Qian, Y.-Z., \& Meyer, B. S. 2007, ApJ, 667, L159

Nomoto, K. 1981, IAU Symp., 93, 295

Nomoto, K. 1984, ApJ, 277, 791

Nomoto, K. 1987, ApJ, 322, 206

Pfahl, E., Rappaport, S., Podsiadlowski, P., et al. 2002, ApJ, 574, 364

Podsiadlowski, Ph., Langer, N., Poelarends, A. J. T., et al. 2004, ApJ, 612, 1044

Poelarends, A. J. T., Herwig, F., Langer, N., \& Heger, A. 2008, ApJ, 675, 614

Ritossa, C., García-Berro, E., \& Iben, I. 1996, ApJ, 460, 489

Ritossa, C., García-Berro, E., \& Iben, I. 1999, ApJ, 515, 381

Sackmann, I.-J. \& Boothroyd, A. I. 1991, ApJ, 366, 529

Siess, L. 2006, A\&A, 448, 717

Siess, L. 2007, A\&A, 476, 893

Siess, L. \& Arnould, M. 2008, A\&A, submitted

Timmes, F. X. \& Woosley, S. E. 1992, ApJ, 396, 649

Vassiliadis, E. \& Wood, P. R. 1993, ApJ, 413, 641

Ventura, P. \& D'Antona, F. 2005, A\&A, 431, 279

\section{Discussion}

KwoK: Observationally, how do we distinguish SAGB from AGB stars?

SiEss: They are more luminous than AGB stars and probably more Helium rich. Asides from that, their surface composition is very similar to that of massive intermediate mass AGB stars that experience hot bottom burning.

WoITKE: The interpulse timescale of your $\mathrm{M}_{\text {initial }}=5 \mathrm{M}_{\odot}$ model matches with $\approx 1000 \mathrm{yrs}$ the timescale of concentric rings observed around PPNe and IRC+10216. Could mass 
loss have reduced the actual mass of IRC +10216 from $5 \mathrm{M}_{\odot} \rightarrow \leqslant 1.5 M_{\odot}$ so that it would match?

WiLlson: Might be possible in exceptional cases, but not for the majority of objects with observed concentric rings.

SIESS: It's possible although it would involve a slightly higher mass. Concerning IRC +10216 it has also been proposed by Nearon et al that the rings were formed by the presence of a companion. 